\title{
Charge transport in organic nanocrystal diodes based on rolled-up robust nanomembrane contacts
}

\author{
Vineeth Kumar Bandari ${ }^{1,2}$, Lakshmi Varadharajan ${ }^{1,2}$, Longqian $\mathrm{Xu}^{1,2}$, \\ Abdur Rehman Jalil ${ }^{1,2}$, Mirunalini Devarajulu ${ }^{1,2}$, Pablo F. Siles ${ }^{* 1,2}$, Feng Zhu ${ }^{* 1,2}$ \\ and Oliver G. Schmidt ${ }^{1,2}$
}

\section{Letter}

Address:

${ }^{1}$ Material Systems for Nanoelectronics, TU Chemnitz, Reichenhainer

Str. 70, 09107 Chemnitz, Germany and ${ }^{2}$ Institute for Integrative Nanosciences, IFW Dresden, Helmholtz Str. 20, 01069 Dresden,

Germany

Email:

Pablo F. Siles* - p.r.siles@ifw-dresden.de; Feng Zhu* -

f.zhu@ifw-dresden.de

* Corresponding author

Keywords:

charge transport; nanomembrane; organic diode; organic nanocrystal; rolled-up nanotechnology

\author{
Beilstein J. Nanotechnol. 2017, 8, 1277-1282. \\ doi:10.3762/bjnano.8.129 \\ Received: 31 January 2017 \\ Accepted: 29 May 2017 \\ Published: 19 June 2017 \\ This article is part of the Thematic Series "Towards molecular \\ spintronics". \\ Guest Editor: G. Salvan \\ (C) 2017 Bandari et al.; licensee Beilstein-Institut. \\ License and terms: see end of document.
}

\begin{abstract}
The investigation of charge transport in organic nanocrystals is essential to understand nanoscale physical properties of organic systems and the development of novel organic nanodevices. In this work, we fabricate organic nanocrystal diodes contacted by rolled-up robust nanomembranes. The organic nanocrystals consist of vanadyl phthalocyanine and copper hexadecafluorophthalocyanine heterojunctions. The temperature dependent charge transport through organic nanocrystals was investigated to reveal the transport properties of ohmic and space-charge-limited current under different conditions, for instance, temperature and bias.
\end{abstract}

\section{Introduction}

Organic semiconductors have been widely applied in developing optoelectronic devices including light-emitting diodes, transistors, sensors [1-3]. Because the large variety of physical properties can be conveniently tuned by manipulating the molecular structure, a clever design of the nanoscale device geometry opens up further intriguing options for novel applications in the fields of optoelectronics and spintronics [4-6]. Organic nanocrystals have drawn much attention in the community because high quality material can be efficiently generated by controlling the nanoscale physical growth and/or chemical syn- thesis process [7-10]. The deposition of organic conjugated molecules is usually realized by thermal evaporation in vacuum. The molecules have van der Waals interaction with an inert substrate which makes them weakly bonded to the substrate [11]. As a result, by controlling the molecules deposition parameters, such as deposition rate and the substrate temperature, it is possible to obtain either amorphous smooth and continuous organic thin films with thicknesses down to a few monolayers or inhomogeneous organic nanocrystals such as organic nanopillars and nanopyramids [11-13]. 
However, the fabrication of organic nanodevices based on nanostructures is a persistent challenge due to difficulties in creating non-destructive contacts [14-16]. Although there have been remarkable advances in the methods for vertically contacting a variety of organic thin films, one of the main challenges to perform such fabrication still lies in the preparation of reliable vertical junctions [16]. Mainly due to the inhomogeneous distribution of nanostructures on the substrate surface, the metallic atoms can inter-diffuse and produce unwanted shortcircuit junctions during the contact formation [14]. Thus, the fabrication of a reliable robust top contact is expected to solve this tedious problem. Part of the authors of this report have developed a novel 'rolled-up nanotechnology' to tackle this challenge [17-19]. By this method, strained nanomembranes are released from a substrate surface and the elastic relaxation of the built-in strain gradient triggers a self-rolling process of the nanomembranes. The strained nanomembranes roll-up into full microtubes and finally land on top of the organic nanostructures, e.g., self-assembled monolayers and organic nanopyramids $[17,19]$. Compared to other 'soft' contact methods developed recently, including chemical binding [20,21], indirect evaporation [22,23], 'ready-made' approaches [24,25], and robust mechanical contacts [26-29], the rolled-up nanotechnology provides the precise positioned electrodes and high fabrication yield of array devices, and does not require the chemical modification of functional organic layers. Furthermore, the candidate materials for rolled-up nanomembranes are metals, ferromagnetic layers, oxides, and complex materials, of which the various properties of thin solid films, e.g., work function and magnetic properties, can be utilized to develop novel functional organic devices [30,31]. In our previous report, organic nanocrystal diodes have been successfully developed, in which rolled-up nanomembranes provide robust contacts to fully unleash the advantages of organic nanocrystals for sensing gas molecules [19]. Apart from the demonstration of functional nanodevices, the investigation and understanding of charge transport mechanisms across the organic nanostructure is a key topic nowadays for developing and optimizing novel nanostructured devices $[8,9,32,33]$.

In this work, we fabricate organic nanocrystal diodes sandwiched between flat metal electrode and rolled-up nanomembrane electrode contacts. The nanocrystals consist of vanadyl phthalocyanine (VOPc) and copper hexadecafluorophthalocyanine $\left(\mathrm{F}_{16} \mathrm{CuPc}\right)$ heterojunctions. The temperature dependent current-voltage behaviors were investigated to unveil the charge transport properties of the nanocrystals. As most of the well-studied charge transport systems are based on planar or vertical bulky organic thin-film devices [34], the conduction mechanism in this report will provide a helpful insight into the charge transport in nanoscale systems.

\section{Results and Discussion}

The fabrication protocol of the organic nanocrystal diodes is the same as in our previous reports [19]. The fabrication yield of the devices contacted by rolled-up electrodes on the single chip can achieve more than $95 \%$ owing to the reliable parallel nanofabrication when the whole process is carefully performed. To study the charge transport properties of the crystalline heterojunction nanopyramids, three kinds of organic nanopyramids were grown on well-defined bottom $\mathrm{Au}$ finger electrodes (Au mesa), i.e., pure VOPc $(10 \mathrm{~nm}), \mathrm{F}_{16} \mathrm{CuPc}(1 \mathrm{~nm}) / \mathrm{VOPc}$ $(9 \mathrm{~nm})$ and $\mathrm{F}_{16} \mathrm{CuPc}(1 \mathrm{~nm}) / \operatorname{VOPc}(8 \mathrm{~nm}) / \mathrm{F}_{16} \mathrm{CuPc}(1 \mathrm{~nm})$. The thicknesses are the nominal values detected by the thickness monitor. However, the deposited molecules form inhomogeneous nanopyramids during growth. The heights of the nanopyramids range between $50-100 \mathrm{~nm}$, as observed from atomic force microscopy (AFM) measurements. Figure 1 shows the device configuration and molecular structures. After formation of organic nanopyramids, the rolled-up Au tube electrodes land on top and form reliable contacts. The geometric contacting area between organic and finger electrodes is estimated by considering the circumference of the tube electrode and the width of the mesa electrode, which is on average about $8 \mu \mathrm{m}^{2}$. An uncertainty concerning the effective electrical contact to the tube electrode remains, because the total number of current pathways across the crystalline nanopyramids is experimentally difficult to determine. The morphology properties of organic layer will determine the effective contact area to a great extent. For instance, a smooth organic layer or self-assembly monolayer has larger contact area when the tube lands on top, while nanopyramid geometry restricts the contact area only within the limited peaks' surface which can touch the tube. Due to geometry deviation of single nanopyramid structure, it is quite challenging to precisely predict how the single tube surface contacts with nanopyramids and calculate the contact area. As a compromise, in this report the transport properties will be deducted based on the electrical current through device, instead of the intrinsic conductivity of single organic nanostructures.

To study the charge transport properties of the crystalline nanopyramids, an electrical characterization is performed by measuring the current-voltage $(I-V)$ characteristics. As shown in Figure 2a, the strong charge transfer (CT) between VOPc and $\mathrm{F}_{16} \mathrm{CuPc}$ causes the heterojunction nanopyramids with double $\mathrm{F}_{16} \mathrm{CuPc}$ buffer layers to experience much-improved charge injection/transport under the positive bias, and a smaller open voltage compared to the devices consisting of pure VOPc and single $\mathrm{F}_{16} \mathrm{CuPc}$ buffer layer. As introduced in our previous report [19], the Schottky barrier due to the poor electric contact between the organic material and the electrodes will restrain the charge transport in the diodes consisting of pure VOPc or organic nanostructure with single $\mathrm{F}_{16} \mathrm{CuPc}$ buffer layer. In this 


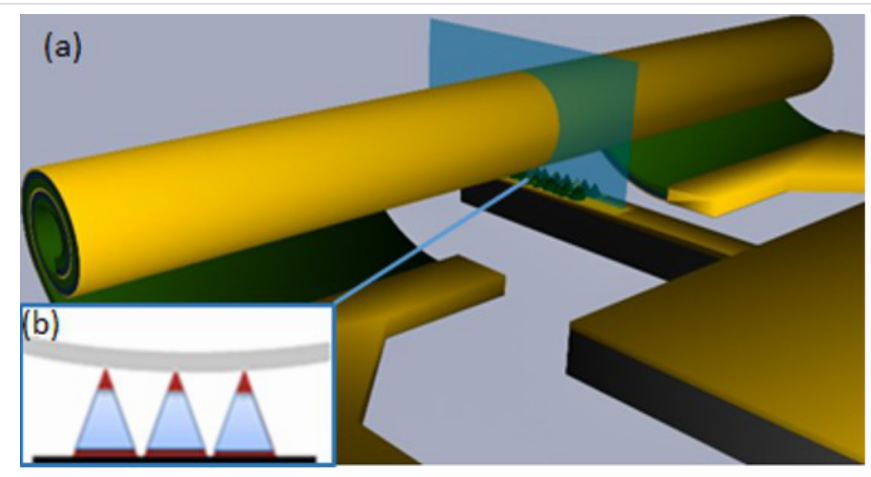

(c)

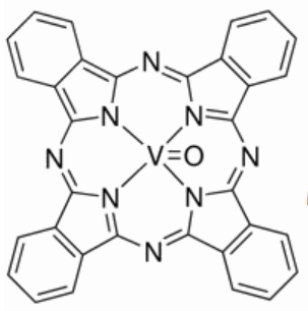

VOPc

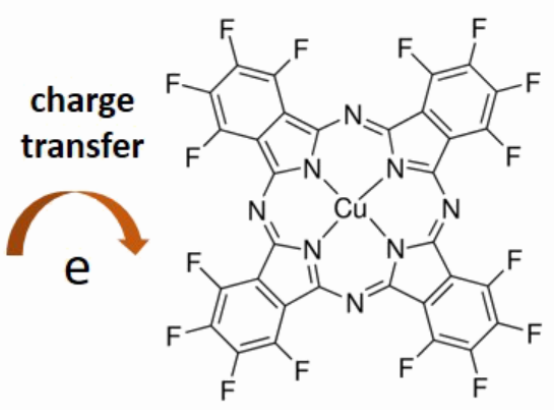

$\mathrm{F}_{16} \mathrm{CuPc}$ (d)

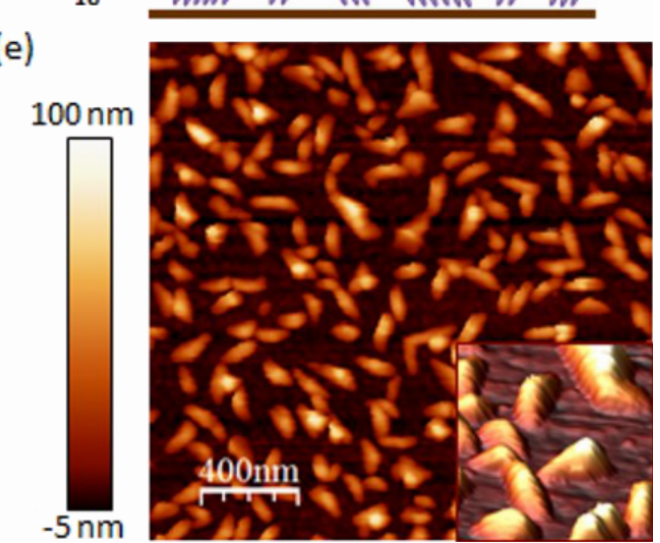

Figure 1: (a) Schematic picture of organic nanocrystal diode with rolled-up contact electrode. (b) Schematic picture of the vertical junction with the nanopyramid sandwiched between the Au mesa electrode and Au tube electrode. (c) Molecular structures of VOPC and $F_{16}$ CuPc. (d) Illustration of the $\mathrm{F}_{16} \mathrm{CuPc} / \mathrm{VOP} / \mathrm{F}_{16} \mathrm{CuPc}$ nanopyramid. (e) AFM topography image of the $\mathrm{F}_{16} \mathrm{CuPc} / \mathrm{VOP} / \mathrm{F}_{16} \mathrm{CuPc}$ nanostructures.
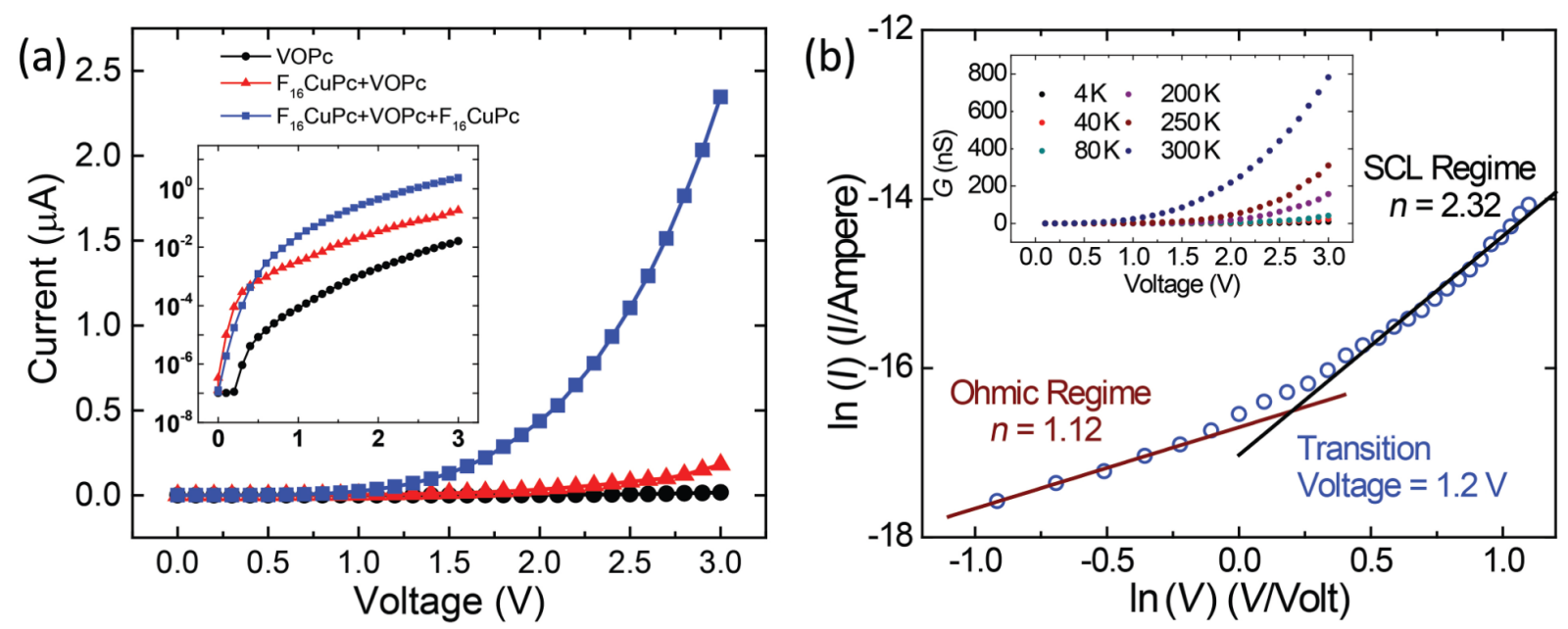

Figure 2: (a) $I-V$ characteristics of three kinds of nanopyramid structures: pure VOPc (black), $\mathrm{F}_{16} \mathrm{CuPc} / \mathrm{VOPc}(\mathrm{red})$ and $\mathrm{F}_{16} \mathrm{CuPc} / \mathrm{VOP} / \mathrm{F}_{16} \mathrm{CuPc}$ (blue), (b) $\ln (I)-\ln (V)$ plot showing the transition of transport regimes from ohmic to SCL.

report, we will focus on the investigation of charge transport in the $\mathrm{F}_{16} \mathrm{CuPc} / \mathrm{VOPc} / \mathrm{F}_{16} \mathrm{CuPc}$ organic nanopyramid diode by applying a forward bias to the tube electrode.

With the $\mathrm{F}_{16} \mathrm{CuPc}$ buffer layers the electrical contacts between the organic nanopyramids and the tube electrode are ohmic at room temperature. A typical plot of the natural logarithm of the current versus natural logarithm of the voltage $(\ln (I)-\ln (V))$ is shown in Figure 2b, which allows us to determine two transport regimes. The curve has a slope of $n=1.12$ for low bias and a slope of $n=2.32$ for a high bias. The transition voltage is about $1.2 \mathrm{~V}$. According to the Mott-Gurney Law the transport can be 
regarded as ohmic in the low bias regime and space-chargelimited (SCL) in the high bias regime [35], which agrees with our previous report [19,36].

To assess the transport process of the vertical nanopyramid device based on the $\mathrm{Au}$ mesa electrode $/ \mathrm{F}_{16} \mathrm{CuPc} / \mathrm{VOPc} /$ $\mathrm{F}_{16} \mathrm{CuPc} / \mathrm{Au}$ tube electrode, $I-V$ measurements at different temperatures were performed, as shown in Figure 3a. Similar to the current-voltage characteristics at room temperature, the current under forward bias remains dominant also at lower temperatures. By plotting the temperature dependent current behavior $(\ln (I)-1000 / T)$ under different bias we obtain two distinct regions with different slopes the change-over of which occur at around $125 \mathrm{~K}$, as shown in Figure $3 \mathrm{~b}$. The curves for $T>125 \mathrm{~K}$ (left part) show more pronounced temperature dependence, which indicates that thermal activation plays an important role during the transport [37]. Both the left and right regions of Figure $3 b$ are well-described by the classical Arrhenius relation.

$$
I_{0}=A_{1} S V \cdot \exp \frac{-E_{\mathrm{a}}}{k_{\mathrm{B}} T}
$$

where $E_{\mathrm{a}}$ is the activation energy, $k_{\mathrm{B}}$ the Boltzmann's constant, $A_{1}$ the pre-exponential factor, and $S$ the estimated contact area.

The occurrence of two thermally activated regions can be explained as the following: for lower temperature, the CT effect between VOPc and $\mathrm{F}_{16} \mathrm{CuPc}$ becomes weak and the contact between the organic material and the electrodes lose their ohmic contact properties. Thus, the current is mainly governed by the Schottky barrier. For higher temperature and higher bias, the current depends on the charge transport ability which is limited by the hopping mobility. For higher temperature and lower bias, the ohmic current is dominated by the temperature dependent carrier density which is provided by the CT between VOPc and $\mathrm{F}_{16} \mathrm{CuPc}$. In the left part of the curves which are subject to higher activation energy, the activation energy $E_{a}$ is plotted for each bias voltage.

As shown in Figure $3 \mathrm{c}$, the $E_{\mathrm{a}}$ as a function of applied voltage $V$ can provide more direct information about the thermal activated transport progress above $125 \mathrm{~K}$. The plotted region can be divided into three regimes. Region A under low bias corresponds to the ohmic conduction region. The amount of current is mainly determined by the density of carriers. With low electric field, the injected mobile carriers are much lower than the carriers generated from $\mathrm{CT}$ effect between VOPc and $\mathrm{F}_{16} \mathrm{CuPc}$, therefore, the current is mainly due to the movement of CT mobile carriers, of which the density is subject to thermal activation. The activation energy $E_{\mathrm{a}}$ is almost constant with decreasing voltage, and calculated to be about $0.41 \mathrm{eV}$ for the
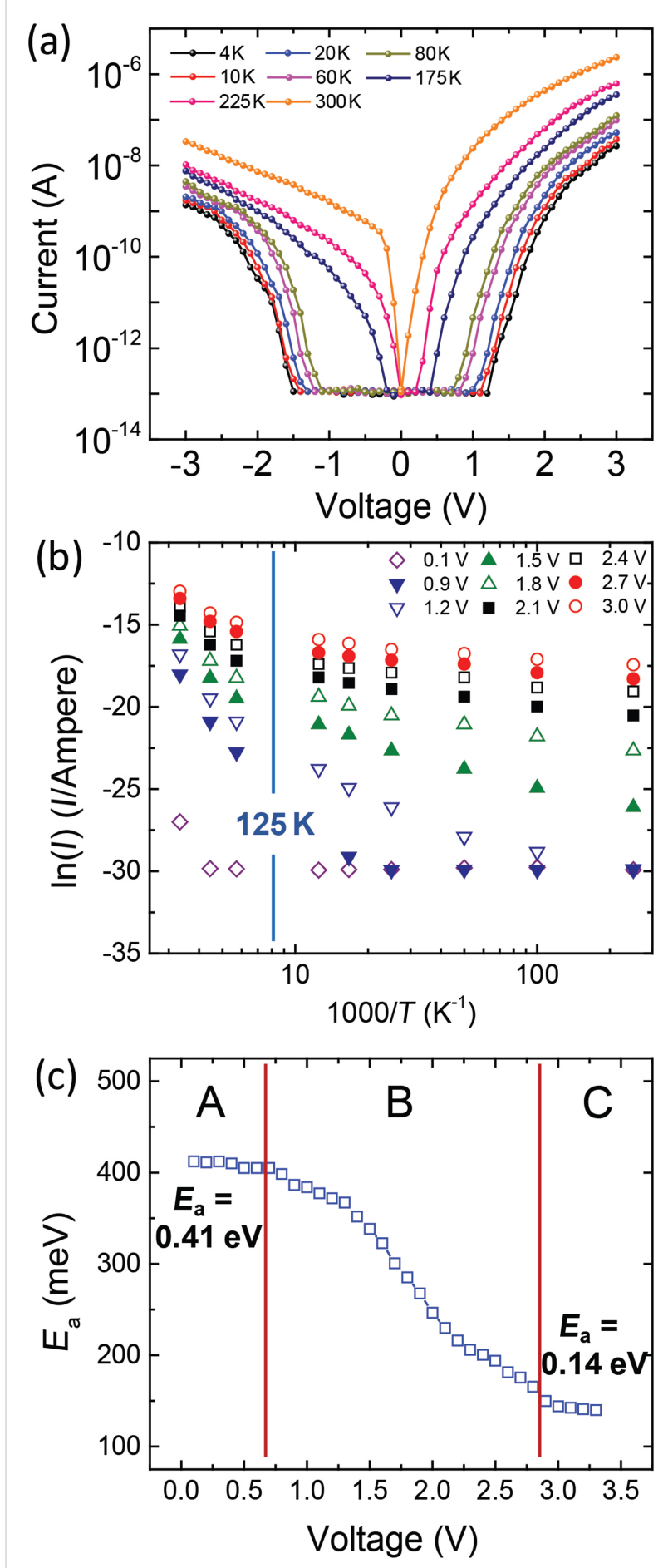

Figure 3: (a) Current-voltage characteristics of Au/ $\mathrm{F}_{16} \mathrm{CuPc} / \mathrm{VOP} /$ $\mathrm{F}_{16} \mathrm{CuPc} / \mathrm{Au}$ diode as a function of temperature. (b) Current-temperature characteristics at different voltages. (c) Applied voltage dependence of thermal activation energy.

voltages below $0.7 \mathrm{~V}$, which is regarded as the CT energy between VOPc and $\mathrm{F}_{16} \mathrm{CuPc}$. Region $\mathrm{C}$ under high bias conditions corresponds to the complete SCL region. As discussed above, the amount of current is mainly determined by the 
hopping mobility as the amount of injected carriers are much higher than the carriers generated from CT. The activation energy $E_{\mathrm{a}}$ is almost constant with increasing voltage, and calculated to be about $0.14 \mathrm{eV}$ for the voltages above $2.8 \mathrm{~V}$, which is regarded as the activation energy for the carrier hopping transport $[34,35,38]$. Region $\mathrm{B}$ is the transition region between ohmic and SCL current. Here, the activation energy decreases due to the increase of the charge carrier injection with increasing bias, while the hopping motion in nanocrystals gradually dominates the charge transport. This corresponds to the $I-V$ trace in Figure $2 b$, which shows a smooth transition from ohmic to SCL. As shown in the upper inset of Figure $2 b$, the voltage dependence of the conductance $(G)$ demonstrates that with increasing bias the conductance increases non-linearly and with decreasing temperature the conductance decreases correspondingly. It is worthy to compare here with the diodes consisting of self-assembly monolayer contacted with rolled-up tube electrodes, which is previously reported by some of the authors of this contribution [17]. The transport in such diode is subject to tunneling and field emission mechanisms due to the ultra-thin smooth film, while in the present report the thermal activated transport via bulk nanostructures dominates the transport progress.

\section{Conclusion}

In summary, in this work we investigated the charge transport in $\mathrm{F}_{16} \mathrm{CuPc} / \mathrm{VOPc} / \mathrm{F}_{16} \mathrm{CuPc}$ organic nanocrystal diodes, which are contacted by robust metallic rolled-up nanomembranes. The temperature dependent measurement results demonstrate in the temperature region above $125 \mathrm{~K}$ carrier injection, and the hopping mechanism dominates the transport through the organic nanocrystals. These conclusions prove that with the assistance of the charge transfer effect, the soft yet robust contacts generated from rolled-up nanotechnology provide an efficient route for fabricating reliable organic nanocrystal electronic and spintronic devices.

\section{Acknowledgements}

The authors acknowledge Paul Plocica and Eric Pankenin for their technical support. This research was financially supported by the Deutsche Forschungsgemeinschaft DFG Research Unit 1154 "Towards Molecular Spintronics" and Europäischen Sozialfonds (ESF).

\section{References}

1. Reineke, S.; Lindner, F.; Schwartz, G.; Seidler, N.; Walzer, K.; Lüssem, B.; Leo, K. Nature 2009, 459, 234-238. doi:10.1038/nature08003

2. McCarthy, M. A.; Liu, B.; Donoghue, E. P.; Kravchenko, I.; Kim, D. Y.; So, F.; Rinzler, A. G. Science 2011, 332, 570-573. doi:10.1126/science.1203052
3. Di, C.-a.; Zhang, F.; Zhu, D. Adv. Mater. 2013, 25, 313-330. doi:10.1002/adma.201201502

4. Sirringhaus, H.; Tessler, N.; Friend, R. H. Science 1998, 280, 1741-1744. doi:10.1126/science.280.5370.1741

5. Forrest, S. R.; Thompson, M. E. Chem. Rev. 2007, 107, 923-925. doi:10.1021/cr0501590

6. Dediu, V. A.; Hueso, L. E.; Bergenti, I.; Taliani, C. Nat. Mater. 2009, 8, 707-716. doi:10.1038/nmat2510

7. Vilan, A.; Shanzer, A.; Cahen, D. Nature 2000, 404, 166-168. doi:10.1038/35004539

8. Song, H.; Reed, M. A.; Lee, T. Adv. Mater. 2011, 23, 1583-1608. doi:10.1002/adma.201004291

9. Ratner, M. Nat. Nanotechnol. 2013, 8, 378-381. doi:10.1038/nnano.2013.110

10. Ortmann, F.; Radke, K. S.; Günther, A.; Kasemann, D.; Leo, K.; Cuniberti, G. Adv. Funct. Mater. 2015, 25, 1933-1954. doi:10.1002/adfm.201402334

11. Yang, J.; Yan, D.; Jones, T. S. Chem. Rev. 2015, 115, 5570-5603. doi:10.1021/acs.chemrev.5b00142

12. Forrest, S. R. Chem. Rev. 1997, 97, 1793-1896. doi:10.1021/cr941014o

13. Wang, W.; Chi, L. Acc. Chem. Res. 2012, 45, 1646-1656. doi:10.1021/ar200299w

14. Haick, H.; Cahen, D. Acc. Chem. Res. 2008, 41, 359-366. doi:10.1021/ar700099n

15. Metzger, R. M. Chem. Rev. 2015, 115, 5056-5115. doi:10.1021/cr500459d

16. Xiang, D.; Wang, X.; Jia, C.; Lee, T.; Guo, X. Chem. Rev. 2016, 116, 4318-4440. doi:10.1021/acs.chemrev.5b00680

17. Bof Bufon, C. C.; Arias Espinoza, J. D.; Thurmer, D. J.; Bauer, M.; Deneke, C.; Zschieschang, U.; Klauk, H.; Schmidt, O. G. Nano Lett. 2011, 11, 3727-3733. doi:10.1021/nl201773d

18. Bof Bufon, C. C.; Vervacke, C.; Thurmer, D. J.; Fronk, M.; Salvan, G.; Lindner, S.; Knupfer, M.; Zahn, D. R. T.; Schmidt, O. G. J. Phys. Chem. C 2014, 118, 7272-7279. doi:10.1021/jp409617r

19. Jalil, A. R.; Chang, H.; Bandari, V. K.; Robaschik, P.; Zhang, J.; Siles, P. F.; Li, G.; Bürger, D.; Grimm, D.; Liu, X.; Salvan, G.; Zahn, D. R. T.; Zhu, F.; Wang, H.; Yan, D.; Schmidt, O. G. Adv. Mater. 2016, 28, 2971-2977. doi:10.1002/adma.201506293

20. Loo, Y.-L.; Lang, D. V.; Rogers, J. A.; Hsu, J. W. P. Nano Lett. 2003, 3, 913-917. doi:10.1021/nl034207c

21. Akkerman, H. B.; Blom, P. W. M.; de Leeuw, D. M.; de Boer, B. Nature 2006, 441, 69-72. doi:10.1038/nature04699

22. Haick, H.; Niitsoo, O.; Ghabboun, J.; Cahen, D. J. Phys. Chem. C 2007, 111, 2318-2329. doi:10.1021/jp065357p

23. Bonifas, A. P.; McCreery, R. L. Nat. Nanotechnol. 2010, 5, 612-617. doi:10.1038/nnano.2010.115

24. Magnussen, O. M.; Ocko, B. M.; Deutsch, M.; Regan, M. J.; Pershan, P. S.; Abernathy, D.; Grübel, G.; Legrand, J.-F. Nature 1996, 384, 250-252. doi:10.1038/384250a0

25. Selzer, Y.; Salomon, A.; Cahen, D. J. Am. Chem. Soc. 2002, 124 , 2886-2887. doi:10.1021/ja0177511

26. Reed, M. A.; Zhou, C.; Muller, C. J.; Burgin, T. P.; Tour, J. M. Science 1997, 278, 252-254. doi:10.1126/science.278.5336.252

27. Zhou, C.; Deshpande, M. R.; Reed, M. A.; Jones, L., II; Tour, J. M. Appl. Phys. Lett. 1997, 71, 611-613. doi:10.1063/1.120195

28. Kushmerick, J. G.; Naciri, J.; Yang, J. C.; Shashidhar, R. Nano Lett. 2003, 3, 897-900. doi:10.1021/nl034201n

29. Xiang, D.; Jeong, H.; Lee, T.; Mayer, D. Adv. Mater. 2013, 25 , 4845-4867. doi:10.1002/adma.201301589 
30. Mei, Y.; Solovev, A. A.; Sanchez, S.; Schmidt, O. G. Chem. Soc. Rev. 2011, 40, 2109-2119. doi:10.1039/c0cs00078g

31. Streubel, R.; Lee, J.; Makarov, D.; Im, M.-Y.; Karnaushenko, D.; Han, L.; Schäfer, R.; Fischer, P.; Kim, S.-K.; Schmidt, O. G. Adv. Mater. 2014, 26, 316-323. doi:10.1002/adma.201303003

32. de Silva, A. P.; Uchiyama, S. Nat. Nanotechnol. 2007, 2, 399-410. doi:10.1038/nnano.2007.188

33. Lörtscher, E. Nat. Nanotechnol. 2013, 8, 381-384 doi:10.1038/nnano.2013.105

34. Coropceanu, V.; Cornil, J.; da Silva Filho, D. A.; Olivier, Y.; Silbey, R.; Brédas, J.-L. Chem. Rev. 2007, 107, 926-952. doi:10.1021/cr050140x

35. Kao, K. C.; Hwang, W. Electrical Transport in Solids: With particular reference to organic semiconductors; Pergamon: New York, 1981.

36. Zhu, F.; Yang, J.; Song, D.; Li, C.; Yan, D. Appl. Phys. Lett. 2009, 94, 143305. doi:10.1063/1.3118581

37. Selzer, Y.; Cabassi, M. A.; Mayer, T. S.; Allara, D. L.

J. Am. Chem. Soc. 2004, 126, 4052-4053. doi:10.1021/ja039015y

38. Craciun, N. I.; Wildeman, J.; Blom, P. W. M. Phys. Rev. Lett. 2008, 100, 056601. doi:10.1103/PhysRevLett.100.056601

\section{License and Terms}

This is an Open Access article under the terms of the Creative Commons Attribution License (http://creativecommons.org/licenses/by/4.0), which permits unrestricted use, distribution, and reproduction in any medium, provided the original work is properly cited.

The license is subject to the Beilstein Journal of

Nanotechnology terms and conditions:

(http://www.beilstein-journals.org/bjnano)

The definitive version of this article is the electronic one which can be found at:

doi:10.3762/bjnano.8.129 